\title{
Daño renal asociado con componentes monoclonales débiles en pacientes cubanos con gammapatía monoclonal
}

\author{
Renal involvement associated with weak monoclonal \\ components in Cuban patients with monoclonal \\ gammopathy
}

Ivón Howland-Álvarez, ${ }^{1}$ Yolanda Cruz-Gómez, ${ }^{2}$ Jorge G. Zambrano-Mera ${ }^{1}$

\begin{abstract}
Resumen
OBJETIVO: Analizar la relación entre las bandas monoclonales detectadas en la electroforesis de proteínas séricas y el daño renal.

MATERIALES Y MÉTODOS: Estudio observacional, descriptivo y retrospectivo efectuado a partir de la revisión de los resultados de electroforesis de proteínas efectuado en el Laboratorio de Diagnóstico Clínico del Centro de Investigaciones Médico-Quirúrgicas de La Habana, Cuba, entre enero de 2010 y diciembre de 2016. Criterios de inclusión: pacientes con componente monoclonal detectado en la electroforesis y confirmado por inmunofijación, y pacientes sin componente monoclonal en la electroforesis de proteínas, pero detectado en la inmunofijación realizada por impresión diagnóstica de mieloma múltiple. Los estudios de electroforesis de proteínas en suero y orina de 24 horas se efectuaron en gel de agarosa, con el sistema automatizado Hydrasys 2 $\left(\right.$ Sebia $\left.{ }^{\circledR}\right)$. Para el análisis estadístico se utilizaron las pruebas de Kolmogorov-Smirnov, $\chi^{2} y$ t de Student. Se consideró estadísticamente significativo el IC95\% $(\alpha=0.05)$.

RESULTADOS: Se registraron 73 pacientes con gammapatías monoclonales. En 17 se reportaron concentraciones de inmunoglobulinas en los límites de referencia. La mayoría de los pacientes tuvo concentración elevada de creatinina en suero (129 \pm $46 \mu \mathrm{mol} / \mathrm{L}$ ), aunque solo $7 / 73$ tenía diagnóstico de insuficiencia renal asociada con el componente monoclonal. La concentración de inmunoglobulinas ( $\lg \mathrm{G}, \lg \mathrm{A}$ e $\lg M)$, según sus valores de referencia, se relacionó con el tipo de componente monoclonal detectado. Se encontró un caso de mieloma IgD, con inicio de insuficiencia renal y estudio de electroforesis de proteínas séricas normal.
\end{abstract}

CONCLUSIONES: La inmunofijación sigue siendo un método útil para establecer el diagnóstico de gammapatías monoclonales, sobre todo cuando la electroforesis de proteínas no sugiera esta alteración y pueda verse afectada la función renal.

PALABRAS CLAVE: Gammapatía monoclonal; electroforesis de proteínas; inmunofijación; insuficiencia renal.

\section{Abstract}

OBJECTIVE: To analyze the relationship between the monoclonal bands detected in serum protein electrophoresis and renal involvement.

MATERIALS AND METHODS: An observational, descriptive, and retrospective study was conducted based on the results of electrophoresis and immunofixation carried out at the Laboratorio de Diagnóstico Clínico del Centro de Investigaciones Médico Quirúrgicas in Havana, Cuba, within the time frame of January 2010 and December 2016. Inclusion criteria: patients with monoclonal component detected in electrophoresis and confirmed through immunofixation and patients with no monoclonal component in protein electrophoresis but detected in immunofixation performed for diagnostic suspicion of multiple myeloma. The protein electrophoresis tests in serum and 24-hour urine were

\footnotetext{
${ }^{1}$ Escuela de Laboratorio Clínico, Facultad de Ciencias de la Salud, de la Universidad Técnica de Manabí, Ecuador. ${ }^{2}$ Laboratorio Clínico, Centro de Investigaciones Médico-Quirúrgicas, La Habana, Cuba.
}

Recibido: abril 2018

Aceptado: julio 2018

Correspondencia Ivón Howland Álvarez ihowlandalvarez@gmail.com

Este artículo debe citarse como Howland-Álvarez I, Cruz-Gómez Y, Zambrano-Mera JG. Daño renal asociado con componentes monoclonales débiles en pacientes cubanos con gammapatía monoclonal. Rev Mex Urol. 2018 julioagosto;78(4):243-253.

DOI: https://doi.org/10.24245/revmexurol.v78i4.2135 
conducted in agarose gel, utilizing the automated Hydrasys 2 system (Sebia ${ }^{\circledR}$ ). The Kolmogorov-Smirnov test, chi-square test, and Student's t test were employed for the statistical analysis and the $95 \% \mathrm{Cl}(\mathrm{a}=0.05)$ was considered statistically significant.

RESULTS: 73 patients with monoclonal gammopathy were registered. Immunoglobulin values were within the reference limits in 17 patients. The majority of patients had elevated serum creatinine levels $(129 \pm 46 \mu \mathrm{mol} / \mathrm{L})$, but only $7 / 73$ had the diagnosis of kidney failure associated with the monoclonal component. Immunoglobulin concentration (IgG, IgA, and IgM), according to their reference values, was related to the type of monoclonal component detected. There was one case of IgD myeloma, with the onset of kidney failure, and normal serum protein electrophoresis.

CONCLUSIONS: Immunofixation continues to be a useful laboratory method for diagnosing monoclonal gammopathy, especially when protein electrophoresis does not suggest that alteration and kidney function can consequently be affected.

KEYWORDS: Monoclonal gammopathy; Protein electrophoresis; Immunofixation; Kidney failure.

\section{ANTECEDENTES}

Las gammapatías monoclonales, o discrasias de células plasmáticas, son enfermedades en las que un clon de linfocitos B productores de anticuerpos (células plasmáticas) evade el control normal de la homeostasis inmunoglobulínica y produce inmunoglobulinas anormales o sus fragmentos. A estas proteínas anómalas se les denomina componentes monoclonales, paraproteínas o proteínas $M$; su incremento en la sangre (excepto en el caso del mieloma múltiple no secretor) se detecta por la aparición de una banda homogénea en el trazado electroforético.

Las células plasmáticas sanas producen inmunoglobulinas o anticuerpos. Cada inmunoglobulina contiene dos cadenas pesadas: gamma (lgG), alfa ( $(\operatorname{gA})$, mu (IgM), delta ( $(\operatorname{gD} \mathrm{D})$ o épsilon (IgE), y dos cadenas ligeras (kappa o lambda). El concepto de monoclonalidad implica que estas células plasmáticas neoplásicas producen un único tipo de cadena pesada o ligera (por ejemplo IgA-kappa, IgG-lambda; cadenas ligeras kappa, etc.) y dejan de generar el resto de inmunoglobulinas normales en cantidades suficientes. La elevada concentración de inmunoglobulinas interfiere con diversas propiedades sanguíneas y el funcionamiento adecuado de los riñones, además de aumentar la susceptibilidad a las infecciones, debido a la deficiencia del resto de los anticuerpos. Incluso la elevada producción de células plasmáticas puede lesionar los huesos, provocar dolor, alteraciones osteolíticas y posibles fracturas. Las gammapatías monoclonales se clasifican en malignas y benignas, e incluyen: gammapatía monoclonal de origen incierto, mieloma múltiple, macroglobulinemia de Waldenström, enfermedad de las cadenas pesadas, amiloidosis primaria y síndromes linfoproliferativos en general. ${ }^{1}$

La evaluación clínica sistemática y el uso de mejores técnicas de electroforesis, en cuanto a especificidad y sensibilidad, hacen que el hallazgo fortuito de alguna gammapatía monoclonal sea cada vez más frecuente y sin relación con cierto contexto clínico que la sugiera. ${ }^{2}$ Diversos autores $^{3-5}$ señalan que de todas las gammapatías monoclonales, cerca de $60 \%$ corresponde a una alteración o gammapatía de origen incierto y ésta se define por la detección de algún componente monoclonal (IgG, IgA o IgM) en suero, menor de $30 \mathrm{~g} / \mathrm{L}$, sin o con pequeñas cantidades de cadenas livianas monoclonales libres en orina (proteinuria inferior a $1 \mathrm{~g} / 24 \mathrm{~h}$ ), ausencia de 
lesiones osteolíticas, anemia, hipercalcemia e insuficiencia renal secundaria al componente monoclonal, además de infiltración de células plasmáticas menor de $10 \%$ en la médula ósea. La prevalencia de gammapatía monoclonal de origen incierto en Estados Unidos se estima en $3.2 \%$ de la población mayor de 50 años, incluso aumenta con la edad, y alcanza una tasa de $10 \%$ en pacientes mayores de 85 años. El riesgo de evolución a gammapatía maligna es de 10, 21 y $26 \%$ a 10,20 y 25 años, respectivamente. El mieloma múltiple es la enfermedad más frecuente, ${ }^{6}$ se trata de una alteración de distribución universal, cuyo conocimiento se remonta a la descripción de Sir Henry Bence Jones. Aunque en términos generales no tiene predilección por áreas geográficas, se ha demostrado mayor incidencia en regiones de Europa y América. ${ }^{7}$ La gammapatía monoclonal de origen incierto constituye una etapa premaligna del mieloma múltiple y el componente monoclonal se considera un marcador tumoral adicional en esta enfermedad. ${ }^{8}$

En la década de 1930 el diagnóstico de gammapatías monoclonales se establecía mediante técnicas de aspirado medular y electroforesis, y a partir de 1953 se complementó con inmunoelectroforesis. ${ }^{9}$ La detección de una banda monoclonal debe estar bien definida, focalizada y posicionada a la misma altura que la banda previsible situada en la pista de referencia. La inmunofijación está indicada en pacientes cuyo proteinograma muestre un componente monoclonal y en quienes no muestre dicho componente en el mismo estudio y se sospeche clínicamente de mieloma múltiple, pues la identificación de una banda monoclonal en la prueba de inmunofijación con un proteinograma normal representa un hallazgo frecuente en sujetos con mielomas no secretores, oligosecretores o de cadenas ligeras. El límite de detección de esta técnica es de $0.2 \mathrm{~g} / \mathrm{L}$ en suero y $0.04 \mathrm{~g} / \mathrm{L}$ en orina. De forma similar, la detección de proteínas en orina, al diagnóstico, se realiza en caso de encontrar previamente el componente monoclonal en suero y ante la sospecha de amiloidosis o mieloma múltiple de cadenas ligeras. ${ }^{3}$

Las gammapatías monoclonales suelen aparecer como un único componente monoclonal, pero en algunos casos se han encontrado dos, incluso tres paraproteínas distintas. El diagnóstico de gammapatía oligoclonal implica la determinación de dos bandas de movilidad electroforética diferentes, que pueden formarse por combinaciones iguales o distintas de cadenas pesadas y ligeras. Hasta la fecha se desconoce si la producción de esas paraproteínas se debe a la coexistencia de un solo clon de células plasmáticas, que posteriormente se diversifican en clones independientes después de un proceso de selección antigénica o si se trata desde el comienzo de la trasformación tumoral de clones diferentes. ${ }^{10}$

Para la interpretación de la electroforesis de proteínas debe tenerse en cuenta que, en algunas muestras de suero, la fracción $\alpha 2$ puede tener un ligero desdoblamiento que depende de la concentración y movilidad de sus componentes proteicos. Además, el fibrinógeno residual se expresa como una banda gamma rápida y la proteína $\mathrm{C}$ reactiva, en un contexto inflamatorio, puede aparecer como una banda gamma media. Las lipoproteínas de alta y baja densidad son moléculas complejas con movilidad electroforética variable, desde la zona $\beta$ hasta la zona $\alpha 2$. Su afectación depende del tiempo de almacenamiento de la muestra, concentración y tratamiento con heparina. ${ }^{11} \mathrm{El}$ hecho de que una gammapatía sea benigna no excluye su capacidad de provocar alguna alteración renal secundaria, por lo que al momento de establecer el diagnóstico de gammapatía monoclonal de origen incierto debe efectuarse un estudio renal que incluya la determinación del sedimento, proteinuria y estimación del filtrado glomerular. 
Los médicos tienden a considerar que cuando la electroforesis de proteínas en suero es normal, no se requieren estudios adicionales para descartar la gammapatía monoclonal; sin embargo, existen pautas internacionales bien establecidas. ${ }^{6}$

La afectación renal es frecuente en pacientes con mieloma sintomático y el mecanismo patogénico principal de la nefropatía relacionada con el mieloma es la precipitación intratubular de proteínas monoclonales secretadas por las células neoplásicas (nefropatía por cilindros). En este caso se requiere la secreción de grandes cantidades de la proteína M para producir una precipitación masiva y la clave patogénica en esta nefropatía se encuentra condicionada por la alta carga y agresividad tumoral. Cuando inicia la enfermedad, aunque persista la sospecha clínica, las bandas monoclonales pueden pasar inadvertidas en las pruebas de electroforesis y dar resultados negativos y asociarse con insuficiencia renal $u$ otras causas que descarten el origen hematológico.

El objetivo de este estudio fue: analizar la relación entre las bandas monoclonales detectadas en la electroforesis de proteínas séricas y el daño renal.

\section{MATERIALES Y MÉTODOS}

Estudio observacional, descriptivo y retrospectivo efectuado a partir de la revisión de los resultados de electroforesis de proteínas obtenidos en el Laboratorio de Diagnóstico Clínico del Centro de Investigaciones Médico-Quirúrgicas de La Habana, Cuba, entre enero de 2010 y diciembre de 2016. Se analizaron los resultados de todas las electroforesis de proteínas con inmunofijaciones positivas. Criterios de inclusión: 1) pacientes con componente monoclonal en la electroforesis confirmado por inmunofijación, además de datos analíticos y clínicos suficientes para establecer el diagnóstico de gammapatía monoclonal y
2) pacientes sin componente monoclonal en la electroforesis de proteínas, pero detectado en la inmunofijación realizada por impresión diagnóstica de mieloma múltiple. Criterios de exclusión: ausencia de datos analíticos y clínicos suficientes para establecer el diagnóstico de la gammapatía monoclonal.

Para el estudio sólo se utilizaron los datos de los componentes monoclonales detectados por primera vez en la mayoría de los pacientes y no se tomaron en cuenta los análisis realizados en el seguimiento de algunos casos.

Los estudios de electroforesis de proteínas en suero y orina de 24 horas se efectuaron en gel de agarosa, en el sistema automatizado Hydrasys 2 $\left(\right.$ Sebia $\left.^{\circledR}\right)$. La concentración de los componentes monoclonales (g/L) se obtuvo automáticamente al introducir los valores de las determinaciones químicas de proteínas totales en el programa de cómputo "Phoresis" del sistema Hydrasys 2.

Las variables analíticas evaluadas fueron: zona o fracción de migración electroforética del componente monoclonal (cualitativa) y su concentración, que en el caso de gammapatías monoclonales, según los criterios diagnósticos, ${ }^{6}$ se consideró: componente monoclonal bajo: < $30 \mathrm{~g} / \mathrm{L}$ y elevado: $\geq 30 \mathrm{~g} / \mathrm{L}$. Como intervalos de referencia para la identificación del componente monoclonal en la electroforesis de proteínas se utilizaron los establecidos por el laboratorio: 7.0$15 \mathrm{~g} / \mathrm{L}$. En cuanto a la creatinina, los valores de referencia en hombres fueron de 62-106 $\mu \mathrm{mol} / \mathrm{L}$ y en mujeres de $44-80 \mu \mathrm{mol} / \mathrm{L}$.

Para comprobar el ajuste de la distribución normal de los datos correspondientes a las variables continuas se empleó la prueba de Kolmogorov-Smirnov. En cuanto a la comparación de porcentajes de las variables discretas se aplicó la prueba de $\chi^{2}$. En el caso de variables cuantitativas, para la comparación de las medias 
entre dos grupos se utilizó la prueba t de Student. Se consideró estadísticamente significativo el IC95\% ( $\alpha=0.05)$. En todos los análisis estadísticos se utilizó el programa SPSS: PASW Statistic 18, versión 18.0.0.

El estudio se llevó a cabo con los principios establecidos en la Declaración de Helsinki. ${ }^{12}$ Se respetó la integridad de los pacientes dentro de la investigación, asegurando la confidencialidad de toda la información personal recogida. El trabajo contó con la aprobación del Comité de Ética de la Facultad de Ciencias de la Salud de la Universidad Técnica de Manabí, Ecuador.

\section{RESULTADOS}

Se registraron 73 pacientes con gammapatía monoclonal, con mayor predominio de mujeres. El promedio de edad fue de $59 \pm 13.5$ años (mediana de 60 años y moda de 72 años). Se incluyeron 3 pacientes pediátricos y el paciente con mayor edad tenía 94 años. En el grupo de 60 a 69 se registró el mayor número de casos. No se encontraron diferencias significativas entre las medias de edad, según el sexo de los pacientes ( $p=0.324 ; p>0.05): 58 \pm 15$ años para las mujeres y $62 \pm 9$ años para los hombres.

Al observar minuciosamente las bandas monoclonales en las electroforesis, se encontró que de los 73 pacientes con componentes monoclonales confirmados por inmunofijación, 32 mostraron una banda monoclonal débil o escasamente visible y en 41 se evidenció como una banda o pico monoclonal bien definido, aunque no existieron diferencias significativas. Figura 1

En 17 de 73 casos con gammapatía monoclonal se reportaron valores de globulinas en la electroforesis en intervalos de referencia. Figura 2

La mayoría de los pacientes tuvo concentración elevada de creatinina en suero $(129 \pm 46$ $\mu \mathrm{mol} / \mathrm{L}$ ), aunque solo 7/73 tenía diagnóstico de insuficiencia renal asociada con el componente monoclonal. Diecisiete $(n=17 / 73)$ pacientes con banda monoclonal débil o resultados normales en la electroforesis mostraron valores elevados de creatinina $(117 \pm 26 \mu \mathrm{mol} / \mathrm{L})$; sin embargo, no estuvieron asociados con el componente

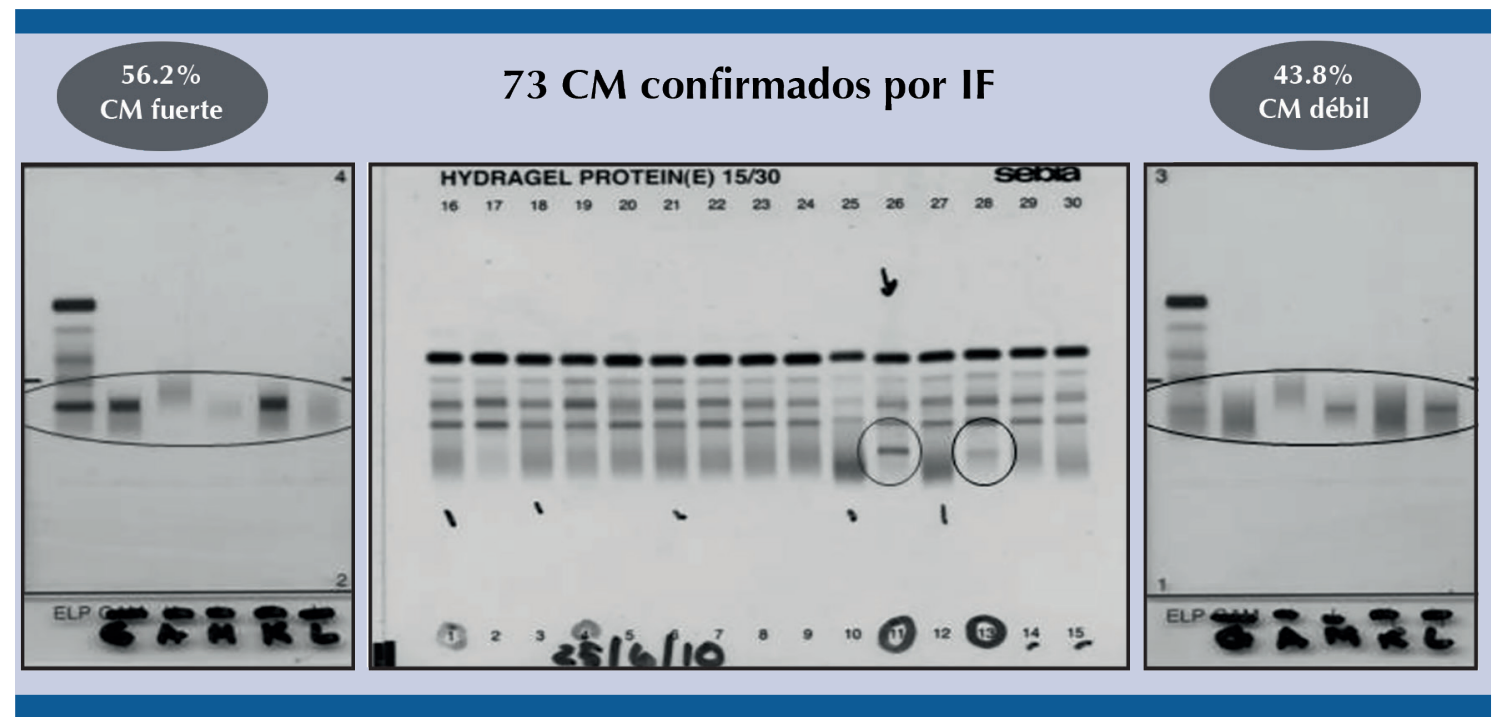

Figura 1. Características de los componentes monoclonales detectados según su intensidad de migración $(p=0.340)$. CM: componente monoclonal; IF: inmunofijación. 


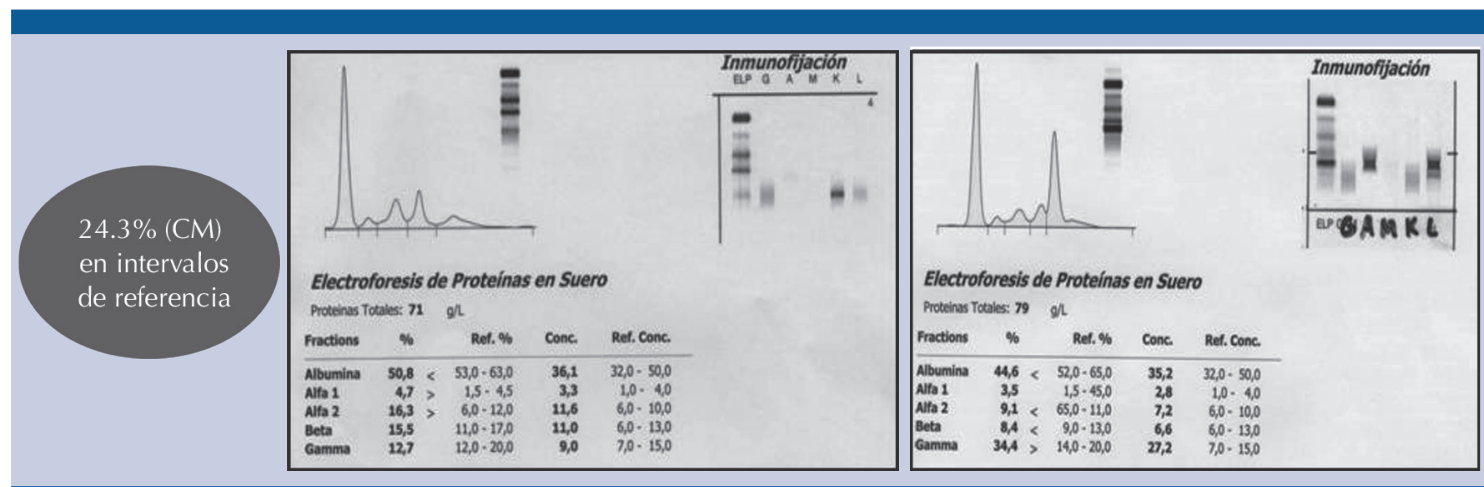

Figura 2. Valores de globulinas en la electroforesis en intervalos de referencia. CO: componente monoclonal.

monoclonal, aunque fueron reportados por el laboratorio como resultados positivos al diagnóstico de gammapatía monoclonal.

El Cuadro 1 describe la distribución de los componentes monoclonales, según su ubicación y las características de migración en las fracciones del electroferograma. Se observó una banda monoclonal en $\alpha 2$-globulinas (que correspondió a una paraproteína de tipo IgA en la inmunofijación), mientras que en 1 caso con componente oligoclonal, diagnosticado con insuficiencia renal crónica, una banda migró a la fracción de $\beta$-globulinas y las otras dos a gammaglobulinas.

La migración puede visualizarse en la Figura 3, donde además se observa la importancia de realizar la inmunofijación en una banda con fuerte expresión, en la región $\alpha 2$-globulinas y el valor de los métodos de diagnóstico alternativos, como la electroforesis de proteínas de alta resolución.

La concentración de inmunoglobulinas (IgG, IgA e IgM), según sus valores de referencia, se relacionó con el tipo de componente monoclonal detectado (Cuadro 2). En 54\% de los componentes monoclonales de tipo IgA y en $50 \%$ de IgM se determinó una concentración por encima de los valores de referencia. Sin embargo, la concentra-
Cuadro 1. Distribución de la migración del componente monoclonal por fracciones en la electroforesis de proteínas

\begin{tabular}{l|c|c|} 
Migración & $\mathbf{n}$ & $\%$ \\
\hline Alfa2 & 1 & 1.4 \\
Beta & 8 & 11 \\
Gamma & 63 & 86.2 \\
Gamma+beta & 1 & 1.4 \\
\hline Total & $\mathbf{7 3}$ & $\mathbf{1 0 0}$ \\
\hline
\end{tabular}

ción de IgG sólo reportó $28 \%$ del componente monoclonal, con resultados más altos que los valores de referencia.

Al analizar cada componente monoclonal se observó que cuando el isotipo implicado de cada inmunoglobulina se incrementó, también disminuyó la concentración de al menos una de las otras (39 del total de casos para IgA y 34 para IgM). Sin embargo, cuando el isotipo implicado fue la IgG, sólo 6 pacientes reportaron disminución de la concentración de alguna de las otras dos inmunoglobulinas de manera simultánea $(p=0.038)$.

En la Figura 4 se muestra un caso de gammapatía monoclonal con diagnóstico de insuficiencia renal, además de los valores de referencia del 
Cuadro 2. Resultado de la cuantificación de inmunoglobulinas en función del componente monoclonal.

\begin{tabular}{l|c|c|c|c|c|}
$\begin{array}{l}\text { Tipo de } \\
\text { componente } \\
\text { monoclonal }\end{array}$ & Total de casos & $\begin{array}{l}\text { Concentración elevada de la } \\
\text { inmunoglobulina respecto del } \\
\text { componente monoclonal total }\end{array}$ & $\begin{array}{c}\text { Inmunoglobulina implicada y disminución } \\
\text { de al menos una de las otras }\end{array}$ \\
\cline { 2 - 6 } & & $\mathbf{n}$ & $\%$ & $\mathbf{n}$ & $\%$ \\
\hline IgG & 51 & 15 & 28.3 & 6 & 11.3 \\
IgA & 12 & 6 & 54.5 & 6 & 54.5 \\
IgM & 10 & 5 & 50 & 5 & 50
\end{tabular}

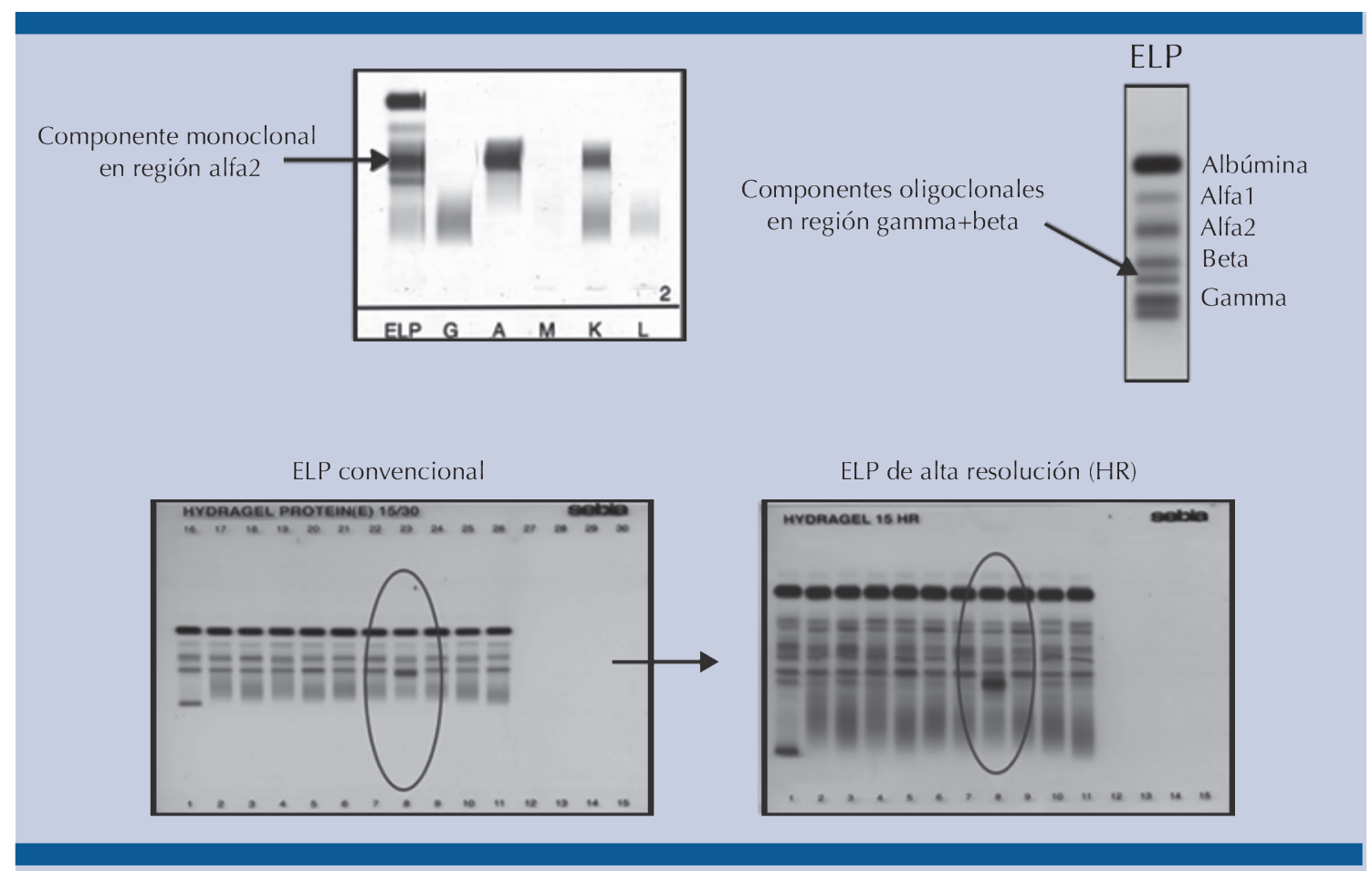

Figura 3. Características de los componentes monoclonales detectados según su zona de migración en la electroforesis, y diferencias entre la electroforesis de proteínas convencional (ELP) y de alta resolución para diferenciar los componentes monoclonales que migran a regiones poco convencionales.

componente monoclonal. En ese paciente la electroforesis de proteínas fue aparentemente normal, pero al observar la inmunofijación convencional con antisueros IgG, IgA e lgM+ kappa y lambda se evidenciaron las cadenas ligeras kappa, sugerentes de mieloma múltiple de Bence Jones. Al realizar nuevamente la inmunofijación con antisueros IgD e lgE se estableció el diagnóstico de mieloma múltiple IgD, mediante estudios de laboratorio, y se comprobó con el estudio en médula por el hematólogo.

\section{DISCUSIÓN}

La interpretación final del proteinograma sérico (análisis primario) y la valoración de las distintas fracciones obtenidas por densitometría, efectuadas por personal con experiencia demostrada en 


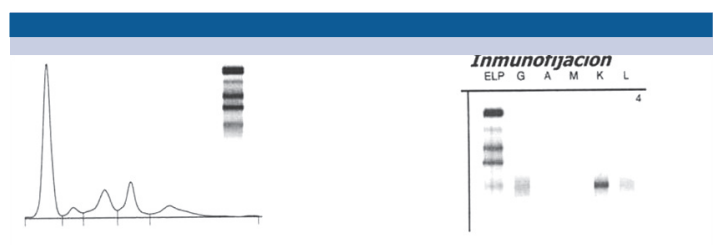

Electroforesis de Proteínas en Suero Proteinas Totales: $\mathbf{7 1} \mathbf{g} / \mathbf{L}$

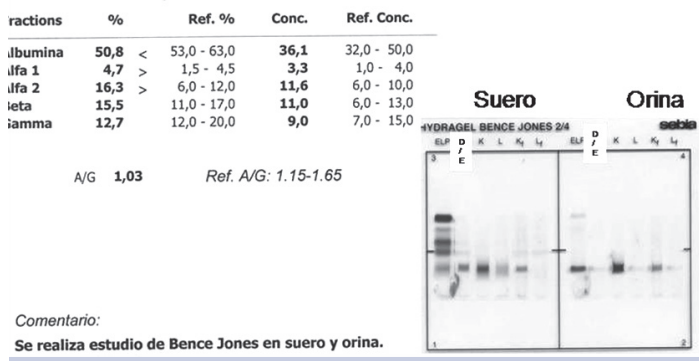

Se realiza estudio de Bence Jones en suero y orina.

Figura 4. Gammapatía monoclonal isotipo IgD con estudio de Bence Jones positivo.

la técnica, en conjunto con la sospecha clínica y otros datos de laboratorio, son factores importantes para decidir la inmunofijación en suero u orina; sin embargo, dicha técnica suele ser costosa. En los últimos años, la incidencia de gammapatías monoclonales ha aumentado debido a la generalización de estudios electroforéticos y a la utilización de sistemas de separación proteica con mayor sensibilidad y especificidad. La resolución final obtenida se encuentra fuertemente influida por el tipo de soporte y las características físicas de la técnica electroforética utilizada $(\mathrm{pH}$, temperatura, voltaje). La sensibilidad analítica del método también se relaciona con el colorante o tinción empleada y su afinidad con las proteínas. ${ }^{13}$

Al igual que en otros estudios, ${ }^{4,8,14,15}$ este ensayo demostró que puede expresarse una banda monoclonal (correspondiente al diagnóstico de gammapatía monoclonal), aunque la concentración total de proteínas séricas, de globulinas beta y gamma, y los valores cuantitativos del compo- nente monoclonal y de las inmunoglobulinas se encuentren dentro de los parámetros normales.

En cuanto a la oligoclonalidad, en algunos casos la proliferación de diversos clones de células B se identifica como varias bandas en el estudio de electroforesis. Cuando en la inmunofijación se observan dos bandas en las zonas correspondientes a las cadenas pesadas y dos en las ligeras (iguales o diferentes en ambos casos), se denomina gammapatía biclonal. La gammapatía oligoclonal se caracteriza por la identificación de múltiples bandas, de uno o más tipos de cadenas pesadas y ligeras. García y su grupo de trabajo $^{10}$ estudiaron 47 pacientes con gammapatía biclonal (3\% de las gammapatías clonales entre los años 1970 y 2011) y encontraron IgG-IgG como la composición más frecuente; además, la gammapatía biclonal de origen indeterminado fue la variante patológica relacionada con mayor frecuencia y en ningún caso evolucionó a malignidad (mediana de seguimiento: 2 años). Al efectuar la segunda determinación observaron que en $81 \%$ de los pacientes había desaparecido al menos uno de los dos componentes monoclonales. En nuestro estudio, el paciente diagnosticado con gammapatía oligoclonal se encontraba en tratamiento con diálisis y su estado era delicado, lo que demuestra que las bandas oligoclonales pueden ensombrecer el pronóstico de la insuficiencia renal secundaria a la enfermedad de base.

El daño renal directamente relacionado con las paraproteínas tiene gran trascendencia clínica, terapéutica y pronóstica; por lo tanto, durante el estudio y seguimiento de las gammapatías monoclonales debe vigilarse de forma sistemática la posibilidad de daño renal, mediante la evaluación de su funcionamiento, el estudio de proteinuria (y albuminuria) y del sedimento urinario. En caso de sospecha de nefropatía es importante el tratamiento multidisciplinario, con estrecha colaboración entre el nefrólogo y el hematólogo. 16,17 
En cuanto a la migración de las bandas monoclonales, la bibliografía describe que el componente monoclonal se expresa como una banda estrecha y densa, y puede encontrarse en la fracción $\gamma o$ $\beta$, incluso en la zona $\alpha 2$ en casos excepcionales. En este estudio los resultados de la migración del componente monoclonal fueron similares a los reportados por Molina y sus colaboradores, ${ }^{18}$ quienes reportaron que $80 \%$ de los pacientes con gammapatía tiene un pico monoclonal en la fracción gamma y $10 \%$ evoluciona con concentraciones normales o hipogammaglobulinemia, que corresponde al diagnóstico de mieloma de Bence Jones.

Los parámetros de referencia de proteínas en orina son similares a los del suero, pero la intensidad relativa de las fracciones puede variar enormemente según la capacidad funcional del riñón. En general, se han definido indicaciones del estudio electroforético e inmunoforético en orina en condiciones como: pico monoclonal en sangre mayor de $15 \mathrm{~g} / \mathrm{L}$, pacientes con diagnóstico clínico de amiloidosis primaria, casos con sospecha de macroglobulinemia de Waldeström o cuando el paciente se ha diagnosticado con mieloma múltiple, para verificar si se excretan inmunoglobulinas o sus fragmentos por la orina y completar el estudio de la enfermedad. ${ }^{16}$

La naturaleza de la inmunoglobulina también es importante en la evolución de la gammapatía monoclonal. Se conoce que la $\lg \mathrm{A}$ e $\lg \mathrm{M}$ son inmunoglobulinas con peor pronóstico y que el riesgo más bajo descrito para gammapatía monoclonal se caracteriza por la identificación de un componente de isotipo IgG. La coexistencia del isotipo IgA representa un signo de mayor invasión medular y de peor pronóstico, pues reportan el mayor número de casos con aumento de sus concentraciones y disminución simultánea de los otros componentes monoclonales. ${ }^{1}$ En los países latinoamericanos se realiza con mayor frecuencia la determinación de la concentración de inmunoglobulinas que la electroforesis por inmunofijación para comprobar el diagnóstico de gammapatía monoclonal. En este estudio se observó que la concentración de alguna de las inmunoglobulinas puede elevarse sin que las otras disminuyan, como ocurre en las gammapatías policlonales; por tanto, esta prueba resulta insuficiente para establecer el diagnóstico de gammapatía monoclonal en sí mismo.

Entre las múltiples causas por las que puede pasar inadvertido un pico monoclonal se encuentran: al inicio de la enfermedad, cuando éste es pequeño (puede coincidir con las áreas de las beta o gammaglobulinas), cuando el pico monoclonal es isotipo $\lg \mathrm{D}$ o $\lg \mathrm{E},{ }^{19}$ pacientes con enfermedad de cadenas pesadas o proteinuria de Bence Jones. En este trabajo se encontró un caso con isotipo IgD kappa en el grupo de mielomas; este componente monoclonal tuvo un pico pequeño (apenas perceptible), similar a lo reportado en la bibliografía para el mieloma de Bence Jones y mieloma múltiple IgD. ${ }^{20,21} \mathrm{El}$ diagnóstico se estableció en una mujer de 48 años, quien inició con insuficiencia renal y se encontraba en tratamiento con diálisis, además de tener antecedentes de diabetes e hipertensión. Sin embargo, después de haber establecido el diagnóstico de mieloma múltiple y recibir tratamiento disminuyó la enfermedad renal de forma considerable. En la mayoría de los pacientes con mieloma múltiple, con isotipo IgD, la insuficiencia renal se identifica oportunamente y, en general, la curación es más rápida que en el resto de los mielomas.

De acuerdo con lo expuesto, el clínico debe identificar las anomalías cualitativas en la electroforesis de proteínas y la inmunofijación, incluso comparar los estudios de los diferentes pacientes. Si bien las bandas localizadas en las gammaglobulinas son fácilmente distinguibles, es importante observar atentamente cualquier aumento de cierta fracción de los componentes. 
Cuando se observa un aumento de las $\beta 2$-globulinas superior al de las $\beta 1$ puede sugerir una IgA monoclonal.

En las pruebas diagnósticas de laboratorio, uno de los problemas más comunes es la disparidad entre muestras idénticas. El trabajo inadecuado puede originar errores en la interpretación de los resultados y tener consecuencias desfavorables en la práctica médica. ${ }^{22}$ Para asegurar la detección de la producción de proteínas monoclonales, el seguimiento debe efectuarse en el mismo laboratorio y con la misma técnica de electroforesis.

Entre las técnicas actuales que ofrecen buenos resultados y son complementarias a la electroforesis de proteínas e inmunofijación en el área de la nefrología se encuentra la cuantificación de las cadenas ligeras libres en suero y el ensayo Hevylite $^{\circledR}$. La determinación de cadenas ligeras libres es un método útil en situaciones como: diagnóstico y seguimiento de pacientes con mielomas no secretores, oligosecretores, de cadenas ligeras, de inmunoglobulinas completas y con amiloidosis; en quienes se pronostica riesgo de evolución a gammapatía monoclonal de origen incierto, mieloma múltiple latente, plasmocitoma óseo y mieloma múltiple de inmunoglobulinas completas, incluso en los casos que no muestran un componente monoclonal por electroforesis (proteinograma) ni por inmunofijación; y en quienes el cociente $\kappa / \lambda$ es normal, sin síntomas clínicos asociados, el estudio permitiría excluir el diagnóstico de gammapatía monoclonal. Hasta la fecha el ensayo Hevylite ${ }^{\circledR}$ no se ha incluido en las guías internacionales para el seguimiento de gammapatías monoclonales. Este ensayo permite calcular cocientes de forma análoga a los de las cadenas ligeras e identificar la proteína monoclonal y diferenciarla de la policlonal de la misma clase. ${ }^{3}$
Tampoco existen parámetros bien establecidos que permitan distinguir a los pacientes con gammapatía monoclonal de origen incierto que evolucionan a estabilidad o malignidad. Solo el control clínico-analítico periódico tiene gran valor en la evolución de estos casos. La detección y vigilancia del componente monoclonal precisa de métodos electroforéticos. Por eso, el laboratorio se encuentra en una situación privilegiada para contribuir con el estudio de la incidencia, distribución y evolución de los pacientes con gammapatía monoclonal. El seguimiento y registro sistemático de los casos es importante para contribuir al conocimiento de las características de este trastorno. La identificación y exploración detallada de las bandas y picos monoclonales débiles, detectados por la sensibilidad de los métodos electroforéticos modernos, permite establecer el diagnóstico y tratamiento oportunos de gammapatía monoclonal maligna o potencialmente maligna.

\section{CONCLUSIONES}

En este estudio se encontraron muestras con componentes monoclonales corroborados por inmunofijación con resultados en la migración de la electroforesis de proteínas dentro de los rangos de referencia (bandas monoclonales débiles) y con valores de creatinina elevados sin estudios renales adicionales que descartaran o comprobaran una insipiente insuficiencia renal. La inmunofijación sigue siendo un método necesario para definir la identificación bioquímica y homogénea de las inmunoglobulinas; por tanto, debe determinarse en suero y orina de 24 horas, cuando el resultado de la electroforesis de proteínas no permita establecer el diagnóstico y persista la sospecha de gammapatía monoclonal y afecte la función renal. La nefropatía concomitante con gammapatías monoclonales puede ser la primera manifestación de la enfermedad; sin embargo, es posible establecer el diagnóstico hematológico a partir de los hallazgos del estudio de la nefropatía. 
Howland-Álvarez I y col. Gammapatía monoclonal

\section{Agradecimientos}

Este trabajo fue financiado por el Centro de Investigaciones Médico-Quirúrgicas (CIMEQ).

\section{Conflicto de interés}

Los autores declaran no tener conflicto de interés.

\section{REFERENCIAS}

1. Llompart I, et al. Estudio retrospectivo de 1.193 componentes monoclonales detectados en Palma de Mallorca. https://doi.org/10.1016/j.labcli.2009.12.004

2. Guía Clinica de Gammapatías Monoclonales, Castilla-León [en línea]. Dirección URL: http://www.sclhh.org/docs/pdf/ Oncoguia\%20GM\%20CyL\%202013\%20final.pdf.

3. Bravo García-Morato M, et al. Guía de laboratorio para el diagnóstico y seguimiento de pacientes con gammapatías monoclonales. https://doi.org/10.1016/j.rce.2015.09.002

4. Alejandre ME, et al. Gammapatía monoclonal de significado incierto: factores de pronóstico, evolución y riesgo. Acta Bioquim Clin Latinoam 2013;47(1):71-84.

5. Howland Al, et al. Caracterización de gammapatías monoclonales. Invest Medicoquir 2012;4(2).

6. Rajkumar SV, et al. International Myeloma Working Group updated criteria for the diagnosis of multiple myeloma. DOI: https://doi.org/10.1016/S1470-2045(14)70442-5

7. Rodríguez $\mathrm{R}$, et al. Caracterización clínica y de laboratorio del mieloma múltiple en el Instituto de Hematología e Inmunología. Rev Cuba Hematol Inmunol Hemoter 2013;29(4):382-97.

8. Kyle RA, et al. Clinical course and prognosis of smoldering (asymptomatic) multiple myeloma. DOI: 10.1056/ NEJMoa070389

9. Howland I, et al. Variables analíticas y clínicas en un estudio de gammapatías monoclonales [en línea]. Dirección URL: http://www.medigraphic.com/pdfs/invmed/cmq-2014/ cmq141g.pdf
10. García-García P. Gammapatías biclonales: estudio retrospectivo de 47 pacientes. DOI: 10.1016/j.rce.2014.07.003

11. Sebia. Electroforesis en gel de agarosa [en línea]. Dirección URL: https://www.sebia.com/es/groupeproduits/ electroforesis-en-gel-de-agarosa

12. Declaración de Helsinki de la AMM - Principios éticos para las investigaciones médicas en seres humanos - WMA The World Medical Association [en línea]. Dirección URL: https://www.wma.net/es/policies-post/declaracion-dehelsinki-de-la-amm-principios-eticos-para-las-investigaciones-medicas-en-seres-humanos/

13. Giraudeaux V. Actuación ante el hallazgo fortuito de una gammapatía monoclonal. Acta Bioquím Clín Latinoam 2004;38(2):207-210.

14. O'Connell TX, et al. Understanding and interpreting serum protein electrophoresis. Am Fam Physician 2005;71(1):105-112.

15. Allué-Dieste $\mathrm{E}$, et al. Gammapatía monoclonal en atención primaria. DOI: 10.1016/j.aprim.2009.06.026

16. Howland I, et al. Gammapatía monoclonal: un diagnóstico a tener en cuenta. Invest Medicoquir 2012;3(2):87-94.

17. Fulladosa X. Nefropatía asociada a gammapatías monoclonales. En: Lorenzo V, López Gómez JM, editores. Nefrología al Día. 2a ed. [en línea]. Dirección URL: http://dev.nefro. elsevier.es/es-monografias-nefrologia-dia-articulo-nefropata-asociada-gammapatas-monoclonales-15

18. Molina MJ, et al. Diagnóstico diferencial de las gammapatías monoclonales. An Med Interna 2006;23(11):546-51.

19. Bovone SN, et al. Mieloma IgE. Dificultades de laboratorio para su tipificación. Medicina (Buenos Aires) 2014;74(6):472-3.

20. Medina $\mathrm{S}$, et al. Marcadores pronósticos en pacientes con gammapatía monoclonal de significado incierto. DOI: 10.1016/j.inmuno.2013.10.001

21. Larson D, et al. Prevalence and monitoring of oligosecretory myeloma. N Engl J Med. 2012;367(6):580-1.

22. Villar-Guimerans $\mathbf{L M}$, et al. Análisis interlaboratorio de parámetros inmunoquímicos, paraproteínas y bandas oligoclonales. Informe técnico del IV Taller de Inmunoquímica de la Sociedad Española de Inmunología. DOI: 10.1016/ S0213-9626(09)70030-7 